\section{The role}

\section{of chronic endometritis in development of infertility in terms of etiological factors}

\subsection{9/bio-up.1.2021.22-27}

Ihor I. Chermak', Volodymyr I. Chermak', Mariia A. Matviann', Mansi Singh ${ }^{4}$

hronic endometritis (CE) is a persistent inflammation of the inner lining of the uterine cavity caused by bacteria such as Enterococcus, Streptococcus, Staphylococcus, Enterobacteriaceae. A majority of CE produces no signs or sometimes mild symptoms, and the prevalence rate of CE is $10-$ $15 \%$. Several studies have shown that it is a condition frequently associated with repeated unexplained implantation failure at in vitro fertilization, recurrent miscarriage, as well as preterm labor. In this study, we reviewed how chronic endometritis plays an In this study, we reviewed how chre, as well as preterm labor important role in of hysteroscopic procedures in the diagnosis of chronic endometritis.

\section{INTRODUCTION}

Chronic endometritis is a disease characterized by the manifestation of such histopathological changes as endometria edema, increased stromal density with leukocyte infiltration in the stroma and glands and one of the most important signs is the presence of plasma cells in the stroma and endometrial glands.

According to various authors, the prevalence of this pathology in the population ranges from 8 to $72 \%$ in women of reproductive age, such a large difference in indicators is explained by differences in diagnostic criteria, difficulties in diagnosis, the presence of asymptomatic course, relatively small experimental groups of patients [1].

22 Risk factors for CE include transferred or persistent infectious diseases of the genitals, intrauterine manipulations (hysteroscopy,

diagnostic curretage, etc.), surgical interventions on the pelvic

1 Bogomolets National Medical University, Department of Obstetrics and Gynecology of the Institute of Postgraduate Education, Kyiv, Ukraine Kyiv Medical University, Department of Obstetrics and Gynecology, Kyiv,

Kyiv Medical University, Department of Pathological Anatomy, Histology

University, Department of Pathological Anat- organs. For example, previous studies have shown that the prevalence of endometritis is about $10-11 \%$ in patients who had a history of hysteroresectoscopy or benign gynecological diseases [3]

There are also studies that indicate the relationship between violation of vaginal environment and the development of $\mathrm{CE}$. For instance, Khan et al. [2] suggests that women with endometritis have higher vaginal $\mathrm{pH}(\geqslant 4.5)$ than in controlled subjects stating that this higher diversity of bacteria' in wom that this higher diversity of bacteria altered vaginal environment. This also altered vaginal environment. This also
suggests that women with endometritis suggests that women with endometritis
could have reduced population of lactic acid producing bacteria mainly Lactobacilli spp. This decrease was also seen in women with bacterial vaginosis, supporting the theory that decreased Lactobacilli level contributes to the development of endometritis.

But the most scientists believe the basis for endometritis is a chronic infection. Cicinelli et al. [11] analyzed 438 cases of hysteroscopically diagnostic CE hysteroscopically diagnostic CE, and reported Most endometrial infections were common bacterial infections (58\%), including Gram-free bacteria, Streptococcus (27\%), E. Coli (11\%), Enterecoccus faealis (14\%) and Ureaplasma urealyticum found in $10 \%$ of cases. Chlamydia was found in $2.7 \%$ of cases [5]

Also in women who could not get pregnant and had a history of recurrent abortions, chronic endometritis as a cause of infertility was $63.3 \%$. Thus women who were treated with antibiotics later had a significantly higher rate of successful pregnancies compared with women who received no treatment that can indicate infectious etiology in favor of CE and among this population one of the most common pathogens was Mycoplasma [6].

In fact, several genes like IGF1, IGF2 and IGFBP1 are also involved in chronic endometritis leading to infertility. IGF1 gene is responsible for endometrial proliferation by mediating estrogen while IGF2 gene is responsible for implantation and invasion of embryo during secretory phase by mediating progesterone. But both these genes are downeguated while gene IGFBP1 is upregulated during chronic endometritis [4]. Gene IGFBP1 will we negative effect on gene IGF1 and IGF2 which will result in infavorable conditions for implantation and then will further result in infertility.

The problem of chronic endometritis is that it cause several complications like infertility, miscarriage, recurrent implantation failure and usually this disease has an asymptomatic course complicates diagnosis.

The association of CE with infertility has been demonstrated in many studies. For example, he prevalence of chronic endometritis in the population of women who had abnormal uterne bleeding and infertility problems according to a retrospective cohort study was $24.4 \%$, the study included 1551 women. Chronic endometritis was diagnosed when plasma cell in endometrial tissue was detected by immunohistochemistry using CD138 epitopes. It was also found that the prevalence of $C E$ in conditions such as recurrent implantation failure $(40.8 \% \cdot \mathrm{P}<0.001)$ (1) endometrial hyperplasia $(50.0 \%, \mathrm{P}<0.05)$ and submucosal fibroid significantly grew by $40.8 \%$, $40.7 \%$ and $50.0 \%$, respectively $[7]$.

Romero et al. reported that $15 \%$ women who underwent IVF had a history of $C E$, and the prevalence of CE among women who had recurrent implantation failure was $42 \%$ [8].

The primary diagnosis of chronic endometritis is based on the patient's history and the presence of risk factors. To confirm the severity

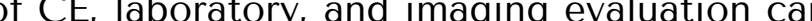
be helpful. The diagnosis may be confirmed by the bio my the be useful to confirm that there is o retained tissue in the uterus. Besides hat hysteroscopy, fluid hysteroscopy, the mmunohistochemical stain is also useful.

Diagnosis of CE is difficult because ususally this pathology has asymptomatic course or non-specific symptoms like abnormal uterine peeding, dysmenorrhea, dyspareunia, pelvic pain, and leucorrhea. and has the presence of asymptomatic course, the lack of clear ultrasonographic signs. Also, despite the fact that the main etiological factor in the occurrence of chronic endometrial pathology is an infectious factor, the identification of $\underset{\mathcal{N}}{\stackrel{0}{\mathcal{O}}}$ 
an infectious agent is quite problematic even in modern conditions. At the same time, the problem of overdiagnosis and the lack of a clearly established etiology leads to inadequate use of antibiotics, which also can have negative
consequences, including exacerbating the problem of antibiotic resistance.

Thus, due to the lack of a clear idea of the etiology, clinical course and nature of morphological changes in this nosology, the result is the lack of pathogenetically determined tactics to restore reproductive function in women with chronic endometritis.

\section{MATERIALS AND METHODS}

We performed biopsy review that focused on the collection of clinical data and the examination of endometrial tissue samples obtained from patients with infertility. Inclusion criteria was clinical diagnosis infertility and chronic endometritis.

A total of 98 endometrial biopsies were analyzed. 98 women referring for infertility had endometrial biopsy in the follicular phase. Mean age was 33.7 years $( \pm 2.3)$

The original diagnoses were made on the basis of the identification of stromal plasma cells.

After the tissue was collected, the samples were immediately immersed in $10 \%$ neutra formaldehid for fixation. Then after ethano gradient dehydratation, the tissue was cleared with xylene. After dehydratation tissue was immersed in paraffin for embedding, and $4 \mu \mathrm{m}$ serial sections were cut. And H\&E routine stain was performed. The assessment of all samples was performed by pathologist using "Olympus CX23" microscope.

The histological diagnosis of chronic 24 endometritis is based on the presence of a
number of criteria that have been used to evaluate endometrial tissue samples:

- an inflammation infiltration that mainly consis of lymphoid elements and this infiltration usually is located focal, near glands and blood vessels and less often - it has diffuse

- focal fibrosis of stroma superficial stromal edema

the presence of a few or even single sroma level plasma cells is enough to settle the diagnosis on chronic endometritis $[9,10]$.

Immunohistochemical evaluation was made using CD-138 antibody (Dako Clone MI15) for all cases that were diagnosed with chronic endometritis during routine microscopica evaluation. Diagnosis chronic endometritis was ma 138 positive plasma cells in the stroma of endometrium.

According to histology and immunohistochemistry results, all cases were divided into three groups $-\# 1-$ patients with clinical diagnosis chronic endometritis which wasn't proven by histological and immunohistochemical studies, \#2 - patient with histological diagnosis which wasn't proven by immunohistochemical study, \#3-- patients with positive immunohistochemistry for chronic endometritis.

Statistical analysis was performed with software IBM SPSS Statistics v. 24. Criteria included mean \pm standard deviation, median, number of positive indicators in group, statica difference were calculated with non-parametric tests such as Mann-Whitney criterion (fo two independent samples) and Kruskal-Wallis I-way ANOVA (for more than two independent samples)

\section{RESULTS}

The mean age of the patients was 33.7 years $( \pm 2.3)$, with a range between 30 and 37 years of age. In 52 women (53\%) histology and immunohistochemical study were negative. Our study found that only $32.6 \%$ of women had chronic endometritis proven by immunohistocemical study. In these groups there were no consistency and differences in age, menstrual irregularities and additional histological findings such as endometrium polyps, abnormalities of uterus etc.
Table 1. Clinical characteristic of study groups.

\begin{tabular}{|c|c|c|c|c|}
\hline \multirow[b]{2}{*}{ Parameter } & \multicolumn{4}{|c|}{ Group } \\
\hline & $\begin{array}{c}\text { Group \#1 } \\
(n=52) \\
53 \%\end{array}$ & $\begin{array}{c}\text { Group \#2 }(n=8) \\
8.1 \%\end{array}$ & $\begin{array}{c}\text { Group \#3 }(\mathrm{n}=32) \\
32.6 \%\end{array}$ & $p$-value \\
\hline $\begin{array}{l}\text { Age, years } \\
\text { Mean } \pm \text { SD } \\
\text { Median }\end{array}$ & $\begin{array}{c}36.2 \\
\pm\end{array}$ & $\begin{array}{c}38.5 \\
\pm\end{array}$ & $\begin{array}{c}34.1 \\
\pm\end{array}$ & $\mathrm{P}=0.71$ \\
\hline $\begin{array}{l}\text { Menstrual irregularities } \\
\mathrm{n}\end{array}$ & $\begin{array}{c}38 \\
(71.6 \%)\end{array}$ & $\begin{array}{c}4 \\
(50 \%)\end{array}$ & $\begin{array}{c}14 \\
(43.7 \%)\end{array}$ & $P=0.26$ \\
\hline $\begin{array}{l}\text { Additional histological } \\
\text { findings such as endo- } \\
\text { metrium polyps, abnor- } \\
\text { malities of uterus etc. }\end{array}$ & $\begin{array}{c}26 \\
(50 \%)\end{array}$ & $\begin{array}{c}3 \\
(37.5 \%)\end{array}$ & $\begin{array}{c}14 \\
(43.7 \%)\end{array}$ & $P=0.63$ \\
\hline
\end{tabular}

Figure 1. Histological slides of negative
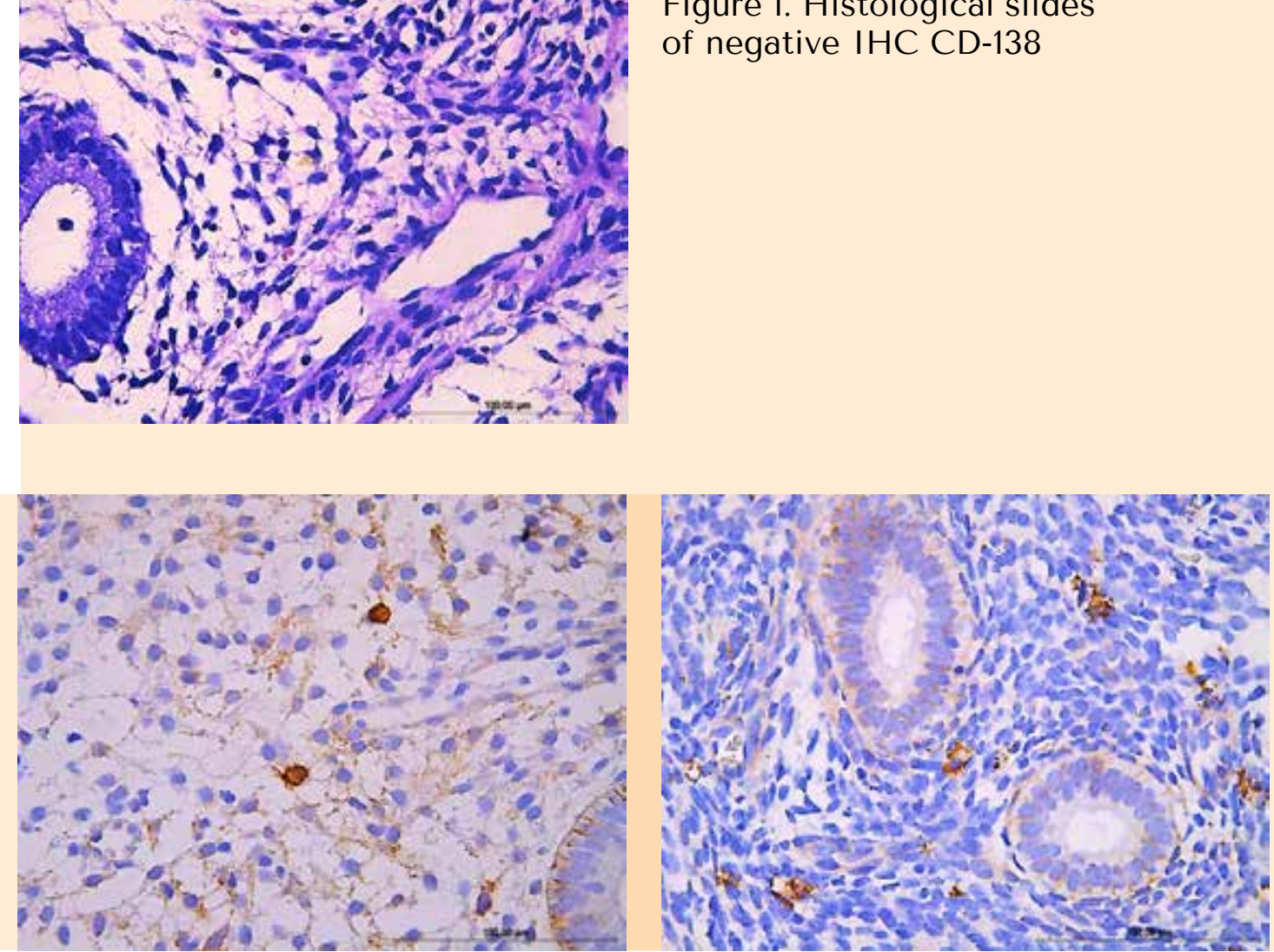

Figure 2. Histological slides of positive IHC CD-138 
We evaluated the structural changes in the endometrium during CE such as edema of stroma superficial fibrosis of stroma and presence of plasma cells, often with impurities of leukocytes and histiocytes. Most often, inflammatory infiltration was localized around glands and blood vessels.

Usually, migration of inflammatory cells, mainly lymphocytes, into the epithelial expulsion of glands and their lumen has been detected.

Structural changes in the endometrium in chronic inflammation were characterized by the presence in the stroma of inflammatory infiltration, represented by a variable number of lymphocytes and plasma cells, often with impurities of leukocytes and histiocytes. Most often, inflammatory infiltration was localized around individual glands and blood vessels. Migration of inflammatory cells, mainly lymphocytes, into the epithelial expulsion of glands and their lumen has been detected. The formation of lymphoid follicles was also often observed not only in the superficial function layer of stroma of the endometrium but in the larea of the basal layer. In the superficin the area of the basal layer. In the supericial areas of the endometrium lymphocytic and also granulocytic infiltration was detected, often directly under the integumentary epithelium which may reflect the infectivity of the contents of the uterine cavity.

In chronic endometritis, reactive changes were observed in the stroma with the proliferation of fibroblast-like, elongated cells and the formation of curls around the glands and blood vessels. Signs of collagenization and even the formation of foci of fibrosis were found in the stroma. Often, areas of endometrial stroma compaction alternated with cytogenic and edematous areas. The hyperemia of the vessels of the microcirculatory tract, fibrosis and thickening of blood vessels walls were natural. The endometrial glands mainly acquired a tu-

${ }_{26}$ bular shape with variable areas of glandula epithelial proliferation.

The histological picture of the endometrium in chronic endometritis often did not correspond with the characteristic morphological features of the glandular and stromal components on specific days of the menstrual cycle and re specific days of the menstral cycles and remetrium
A histological feature of chronic endometritis was the presence of plasma cells in the stroma of the endometrium. These cells wer found diffusely in the stroma of the endometrium and in the lymphoid follicles, even those that were localized in the basal layer of the endometrium. It is known that the identification of plasma cells in the endometrium is the most important sign for the morphological diagnosis of chronic endometritis, In the process of morphological diagnosis there is more evidence of the use of immunohistochemical evichniques using CD138 on MU-1 than histochemical reactions (with methylene green pyronine or acridine orange) [Kondrikov 2008]. The most suitable for morphologic ${ }^{2}$ a agnosis was the material taken on day 8-11 of the menstrual cycle, when the content of immunocompetent cells in the endometrium of healthy women is minimal.

Detection of CD138-positive cells with a membrane pattern of staining in the endometria stroma among the inflammatory infiltrate indicated the presence of morphological signs of chronic endometritis in patients.

\section{CONCLUSIONS}

One of the leading causes of infertility is chronic endometritis. This pathological condition requires comprehensive diagnostics and appropriate treatment. However our study found that nowadays there is a clinical overdiagnosis of chronic endometritis and therefore immunohistochemical study is necessary for chronic endometritis detection. During routine histological study there is also a risk of overdiagnosis although in our study the error is insignificant (only 8 cases). The study has shown that only $32,6 \%$ of women had chronic endometritis proven by immunohistochemica test that once more indicates the presence of overdiagnosis.

The overdiagnosis of this condition is a serious issue that leads to the inadequate antibiotic therapy which can cause the problem of antibiotic resistance. Hence we recommend obligatory immunohistochemical study for clinical diagnosis verification.

\section{REFERENCES}

1. Fuminori Kimura, Akie Takebayashi, Mitsuaki Ishida, Akiko Nakamura, Jun Kitazawa Aina Morimune, Kimiko Hirata, Akimasa Takahashi, Shoko Tsuji, Akiko Takashima, Tsukuru Amano, Shunichiro Tsuji, Tetsuo Ono, Shoji Kaku Review: Chronic endometritis and its effect on reproduction. The journal of Obstetrics and Gynecology Research. 2019.

2. Khan KN, Fujishita A, Kitajima $M$, et al. Intrauterine microbial colonization and occurrence of endometritis in women. 2014;29:2446-56

3. Greenwood SM, Moran JJ. Chronic endometritis. morphologic and clinical observations. Obstet Gynecol. 1981;58:176-184.

4. Ness RB, Soper DE, Holley RL, Peipert J, Randall $H$, Sweet $R L$, et al. Effectiveness of inpatient and outpatient treatment strategies for women with pelvic inflammatory gies for wolts from the Pelvic Inflammadisease. results from the Pelvic InflammatorACH) randomized tial. Am J Obsteth (PEACH) randomized trial. Am J Obstet Gynecol 2002; 186:929-37

5. Cicinelli $E_{1}$ De Ziegler $D$, Nicoletti $R, T i-$ nelli $R$, Saliani N, Resta $L$, et al. Poor reliability of vaginal and endocervical cultures for evaluating microbiology of endometrial cavity in women with chronic endometritis. Gynecol Obstet Invest. 2009;68(2):108115.
6. Cicinelli $E_{1}$ Matteo $M$, Tinelli $R$, Lepera $A$ Alfonso $R$, Indraccolo $U$, Marrocchella $S$, et al. Prevalence of chronic endometritis in repeated unexplained implantation failure and the IVF success rate after antibiotic therapy. Human Reproduction. 2015; 30 $323-30$

7. Dongmei Song, Xiangdong Feng, Qian Zhang, Enlan Xia, Yu Xiao, Wei Xie, Tin Zhiu $L$ i Plevalence and confounders of chronic endometricis in premenopausal chronic endometrinis in premenopasa women with ab ductive failure.

8. Romero R, Espinoza J, Mazor M. Can endometrial infection/inflammation explain implantation failure, spontaneous abortion, and preterm birth after in vitro fertilization? Fertil Steril. 2004;82:799-804.

9. Smith M, Hagerty K A, Skipper B, Bocklage T. Cronic endometritis: a combined histopathologic and clinical rewiew of cases from 2002 to 2007 . Internationat Journal of Gynecologic Pathology 2010; 29: 44-50.

0. Pitsos M, Sturnick J. Heller D. Association pf pathologic diagnoses with clinical findings in chronic endometritis. Journal of Reproductive Medicine 2009; 54 (6): 373-7.

11. Cicinelli E, De Ziegler D, Nicoletti R, Colafiglio G, Saliani N, Resta L, et al. Chronic endometritis: correlation among hysteroscopic, histologic, and bacteriologic findings in a prospective trial with 2190 consecutive office hysteroscopies. Fertil Steri 2008; 89:677-84.

Corresponding author: Mariia A. Matvian, m.matviian@kmu.edu.ua

Manuscript was recieved on 24 October; accepted for publication on 23 December.

CITE AS: IHOR I. CHERMAK ET AL. THE ROLE OF CHRONIC ENDOMETRITIS IN DEVELOPMENT OF INFERTILITY IN TERMS OF ETIOLOGICALFACTORS

BIOMEDICAL UPDATE, ISSUE 1, 202 